\title{
A gene stacking approach leads to engineered plants with highly increased galactan levels in Arabidopsis
}

Vibe M Gondolf ${ }^{1,2,3 \dagger}$, Rhea Stoppel ${ }^{1,2+}$, Berit Ebert ${ }^{1,2,3}$, Carsten Rautengarten ${ }^{1,2}$, April JM Liwanag ${ }^{1,2}$, Dominique Loqué ${ }^{1,2}$ and Henrik $V$ Scheller ${ }^{1,2,4^{*}}$

\begin{abstract}
Background: Engineering of plants with a composition of lignocellulosic biomass that is more suitable for downstream processing is of high interest for next-generation biofuel production. Lignocellulosic biomass contains a high proportion of pentose residues, which are more difficult to convert into fuels than hexoses. Therefore, increasing the hexose/pentose ratio in biomass is one approach for biomass improvement. A genetic engineering approach was used to investigate whether the amount of pectic galactan can be specifically increased in cell walls of Arabidopsis fiber cells, which in turn could provide a potential source of readily fermentable galactose.

Results: First it was tested if overexpression of various plant UDP-glucose 4-epimerases (UGEs) could increase the availability of UDP-galactose and thereby increase the biosynthesis of galactan. Constitutive and tissue-specific expression of a poplar UGE and three Arabidopsis UGEs in Arabidopsis plants could not significantly increase the amount of cell wall bound galactose. We then investigated co-overexpression of AtUGE2 together with the $\beta$-1,4-galactan synthase GalS1. Co-overexpression of AtUGE2 and GalS1 led to over 80\% increase in cell wall galactose levels in Arabidopsis stems, providing evidence that these proteins work synergistically. Furthermore, AtUGE2 and GalS1 overexpression in combination with overexpression of the NST1 master regulator for secondary cell wall biosynthesis resulted in increased thickness of fiber cell walls in addition to the high cell wall galactose levels. Immunofluorescence microscopy confirmed that the increased galactose was present as $\beta-1,4-$ galactan in secondary cell walls.
\end{abstract}

Conclusions: This approach clearly indicates that simultaneous overexpression of AtUGE2 and GalS1 increases the cell wall galactose to much higher levels than can be achieved by overexpressing either one of these proteins alone. Moreover, the increased galactan content in fiber cells while improving the biomass composition had no impact on plant growth and development and hence on the overall biomass amount. Thus, we could show that the gene stacking approach described here is a promising method to engineer advanced feedstocks for biofuel production.

Keywords: Plant cell wall, UDP-glucose 4-epimerase, Galactan, Pectin, Arabidopsis, Populus, Gene stacking, GalS1, NST1, Artificial positive feedback loop

\footnotetext{
* Correspondence: hscheller@lbl.gov

${ }^{\dagger}$ Equal contributors

${ }^{1}$ Feedstocks Division, Joint BioEnergy Institute, Emeryville, California 94608,

USA

${ }^{2}$ Physical Biosciences Division, Lawrence Berkeley National Laboratory,

Berkeley, California 94720, USA

Full list of author information is available at the end of the article
} 


\section{Background}

Plant cell walls are complex structures composed of polysaccharides influencing plant morphology, defense, growth, and signaling. They also constitute the most abundant biomaterial on earth and have the potential to provide a source of cheap sugars for industrial biotechnology. In lignocellulosic biomass, the cell wall polysaccharides comprise mostly cellulose and glucuronoxylan, a hemicellulose, embedded in highly cross-linked lignin polymers, which protect the polysaccharides from chemical and enzymatic degradation. The hemicellulosic fraction is mostly composed of pentoses (such as xylose and arabinose), which unlike hexoses cannot be easily fermented by yeast into fuels. Two main goals of engineering plants with an altered cell wall composition in order to lower costs and improve efficiency of biofuel production is to decrease recalcitrance by decreasing the lignin content or altering the lignin composition $[1,2]$ or to reduce the content of glucuronoxylan and at the same time increasing the content of polysaccharides composed of a larger proportion of fermentable hexoses [3].

$\beta$-1,4-galactan is found as sidechains attached to rhamnogalacturonan I and is generally not highly abundant in lignocellulosic biomass. However, since $\beta-1,4-$ galactan is composed entirely of galactose residues, which can be easily fermented by yeast, an increased content of this polysaccharide would potentially improve the biomass composition for biofuel purposes. In this study we used a genetic engineering approach to specifically increase the amount of $\beta$-1,4-galactan in stem cell walls.

Cell wall polysaccharides are synthesized by glycosyltransferases, which catalyze the formation of glycosidic linkages to form glycosides. During this process monosaccharides from activated sugar substrates are transferred onto glycosyl acceptors. Donor sugars are usually nucleotide sugars, while acceptors can be oligo- or polysaccharides, lipids, proteins, nucleic acids or other small molecules [4]. Recently, the Arabidopsis glycosyltransferase GALACTAN SYNTHASE 1 (GalS1) was shown to be a $\beta-1,4-$ Galactan synthase. Constitutive overexpression of GalS1 in Arabidopsis wild-type plants led to a $50 \%$ increase in cell wall bound galactose in leaves [5]. Loss-of-function mutants in Gals1 or its homologs Gals2 and Gals3 had a larger decrease in cell wall bound galactose in leaves than in stems [5], which suggested that the supply of UDP-galactose might be limiting in stems.

Nucleotide sugars are synthesized by different types of interconverting enzymes such as epimerases, decarboxylases and dehydrogenases. Most of these enzymes are located in the cytosol but some are found within the Golgi lumen [6]. Changes in nucleotide sugar pools can affect the biosynthesis of cell wall polysaccharides, as shown for example for the UDP-glucose dehydrogenase (UGD) double mutant $u g d 2 / u g d 3$, which exhibits significantly reduced cell wall arabinose, xylose, apiose, and galacturonic acid levels [7]. Similarly, the UDP-xylose 4epimerase (UXE) mutant mur4 has a 50\% decrease in cell wall arabinose [8].

The nucleotide sugar UDP-galactose is formed from UDP-glucose by UDP-glucose 4-epimerase (UGE). Five UGE isoenzymes exist in Arabidopsis (AtUGEs), all of which have been functionally characterized in vivo [9-11]. Differences in the expression pattern, kinetics and amino acid sequences of the five AtUGEs suggest that these isoenzymes have an overlapping, but not identical function in plants. UGE-overexpressing plants and knockout mutants have been generated, but specific roles for each of the UGEs could not be unambiguously concluded from those experiments. Only the AtUGE4 knockout mutant ROOT HAIR DEFICIENT 1 (rhd1/UGE4 ${ }^{\text {rhd } 1}$ ) produces a visible phenotype. Roots of uge4 mutants are shorter as compared to the wild type, and the root epidermis cells are swollen due to a defective synthesis of xyloglucan and type II arabinogalactan [10,12,13]. All five AtUGEs can rescue this phenotype when expressed under the control of the constitutive cauliflower mosaic virus $35 \mathrm{~S}$ promoter [9]. In double, triple and quadruple AtUGE mutants, Rösti et al. [10] observed growth defects and cell wall compositional changes that suggested a partial functional overlap of the five UGE isoenzymes. The authors concluded that $A t \mathrm{UGE} 2$ and AtUGE4 affect vegetative growth and cell wall carbohydrate biosynthesis whereas AtUGE1 and $A t$ UGE5 act in stress situations, and AtUGE3 seems to be important for pollen development. Analysis of global coexpression profiles led to the conclusion that AtUGE1 and AtUGE3 are co-expressed with putative trehalose-6phosphate synthase genes, whereas $A t \mathrm{UGE} 2,-4$, and -5 are co-expressed with various known glycosyltransferases and other cell wall biosynthetic enzymes, suggesting that AtUGE1 and AtUGE3 might preferentially act in the UDP-galactose (UDP-Gal) to UDP-glucose (UDP-Glc) direction, while $A t \mathrm{UGE} 2,-4$, and -5 might act in the UDPGlc to UDP-Gal direction in vivo [9].

All UGE isoenzymes can interconvert UDP-Glc and UDP-Gal in vitro, although they show differences in the substrate affinity and reaction requirements $[9,11]$. Interestingly, two isoenzymes, $A t \mathrm{UGE} 1$ and $A t \mathrm{UGE} 3$ have been shown to also interconvert UDP-xylose (UDP-Xyl) and UDP-arabinose (UDP-Ara) [11]. This bifunctionality is reflected in the amino acid sequence of the different AtUGEs. Phylogenetic analysis of UGE homologs in different organisms revealed that UGEs in plants are distributed in two plant-specific clades with $A t \mathrm{UGE} 1$ and $A t \mathrm{UGE} 3$ grouping together in clade I, while AtUGE2, -4 , and -5 group together in clade II (Figure 1). Besides AtUGE1 and $A t \mathrm{UGE} 3$, the pea PsUGE1 has also been shown to interconvert UDP-Xyl and UDP-Ara [11]. PsUGE1 is also 


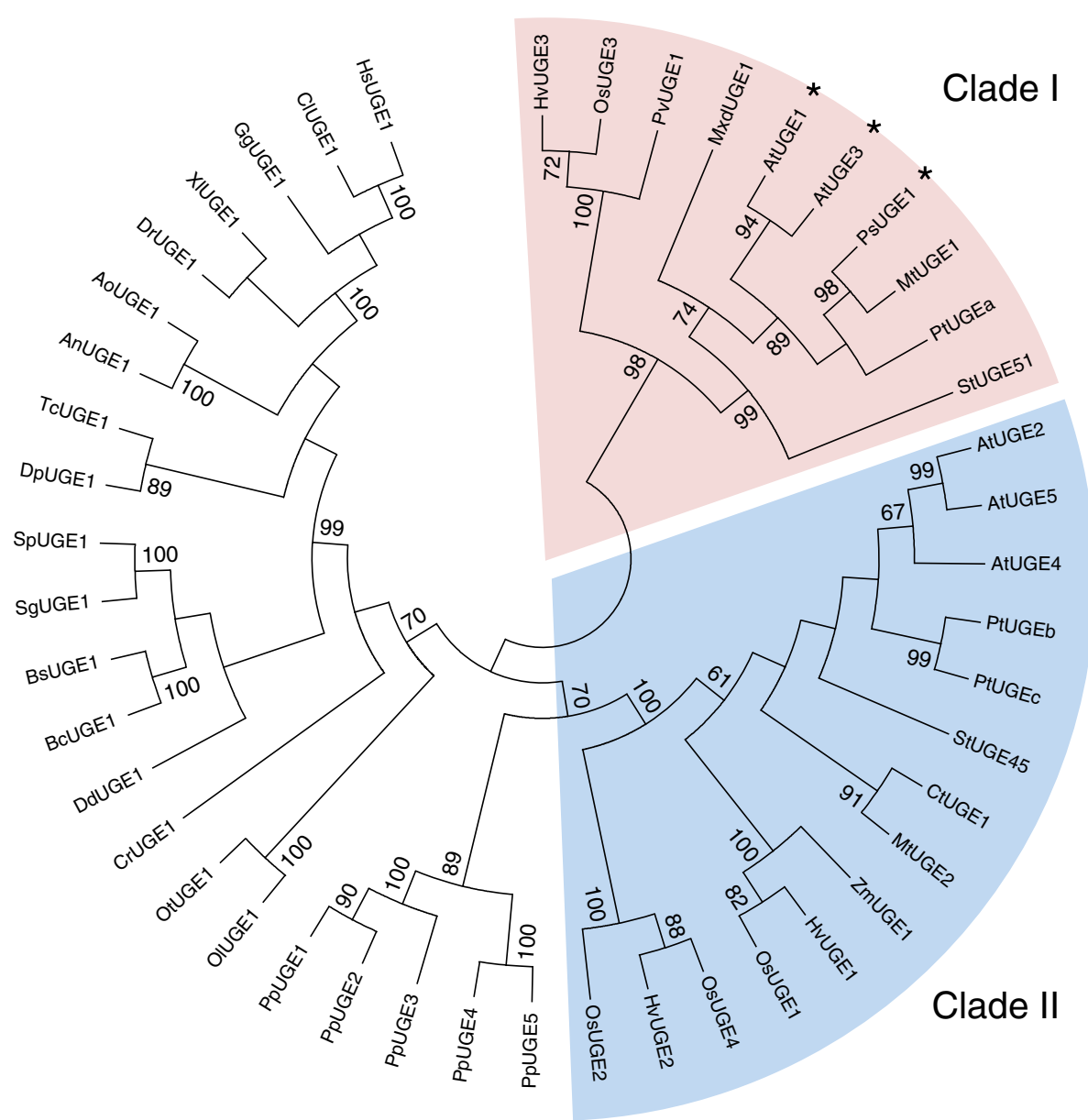

Figure 1 Cladogram of UDP-glucose 4-epimerases. The phylogenetic relationships of UGEs from different organisms are shown. Two vascular plant UGE families described by Kotake et al. [11] are highlighted. UGEs that have documented UDP-Xyl 4-epimerase activity are marked with asterisks. The tree was generated using the Neighbor-Joining method, the bootstrap consensus tree inferred from 1000 replicates (MEGA6 program). Bootstrap values greater than $60 \%$ are indicated. Species and accession numbers are described in the Methods section.

located in UGE clade I, indicating that this clade contains additional bifunctional UGEs. Overexpression of two potato UGEs (StUGE45 and StUGE51) led to an increase of galactose in potato tuber cell walls [14] consistent with the hypothesis that the amount of available UDP-Gal rather than that of galactosyltransferases can be the limiting factor for the accumulation of cell wall galactose.

In the present study, we overexpressed different UGEs from Arabidopsis and poplar, in order to increase the level of cell wall galactose and thereby improve the $\mathrm{C}_{6} / \mathrm{C}_{5}$ sugar ratio. In addition to constitutive overexpression of one poplar and three Arabidopsis UGEs, we expressed one of the Arabidopsis UGEs (AtUGE2) under the control of the secondary cell wall specific promoter pIRX5 together with the master transcription factor NST1. IRX5 is one of the catalytic subunits of the cellulose synthase complex in secondary cell walls [15] and its expression is induced by the transcription factor NST1 [16]. Expression of NST1 under the pIRX5 promoter has been shown to create a transcriptional positive feedback loop enhancing overall expression of secondary cell wall biosynthesis genes and thereby increasing secondary cell wall deposition in fiber cells [2]. Finally, constitutive and fiber-specific promoter AtUGE2 constructs were expressed in the background of transgenic plants overexpressing the galactan synthase GalS1 [5].

While expression of any of the four UGEs alone did not alter the galactose content significantly, regardless of the promoter used, co-overexpression of $A t \mathrm{UGE} 2$ and GalS1 led to an increase in the cell wall galactose content of stems of up to $80 \%$.

\section{Results and discussion}

\section{Populus trichocarpa UGEc is bifunctional in vitro}

In order to compare the performance of different UGEs for the engineering purpose, we first cloned a UGE from poplar since it might be preferable to use a poplar gene for the ultimate translation on the engineering approach to a biofuel crop such as poplar. Out of the UGEs 
encoded in the Populus trichocarpa genome, we selected one, referred to as PtUGEc (XP_002299469, POPTR_0001s10700g). Due to its higher sequence similarity to the non-bifunctional $A t \mathrm{UGEs}$ of Clade II that preferentially act in interconverting UDP-Glc to UDPGal (Figure 1), PtUGEc seemed a good candidate for generating higher amounts of galactan. The in vitro activity of purified His-PtUGEc (Figure 2A) was tested in reactions with different nucleotide sugars (UDP-Glc, UDP-Gal, UDP-Xyl, UDP-Ara). Proportions of UDPsugars after incubation for 30 min show that the $P t \mathrm{U}$ GEc enzyme is bifunctional, interconverting UDP-Glc

\section{A}

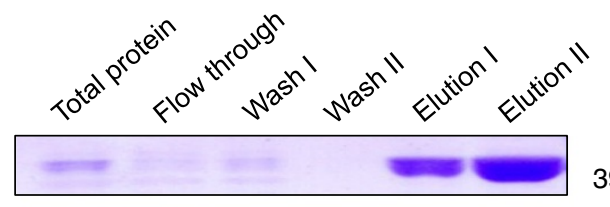

$39 \mathrm{kDa}$
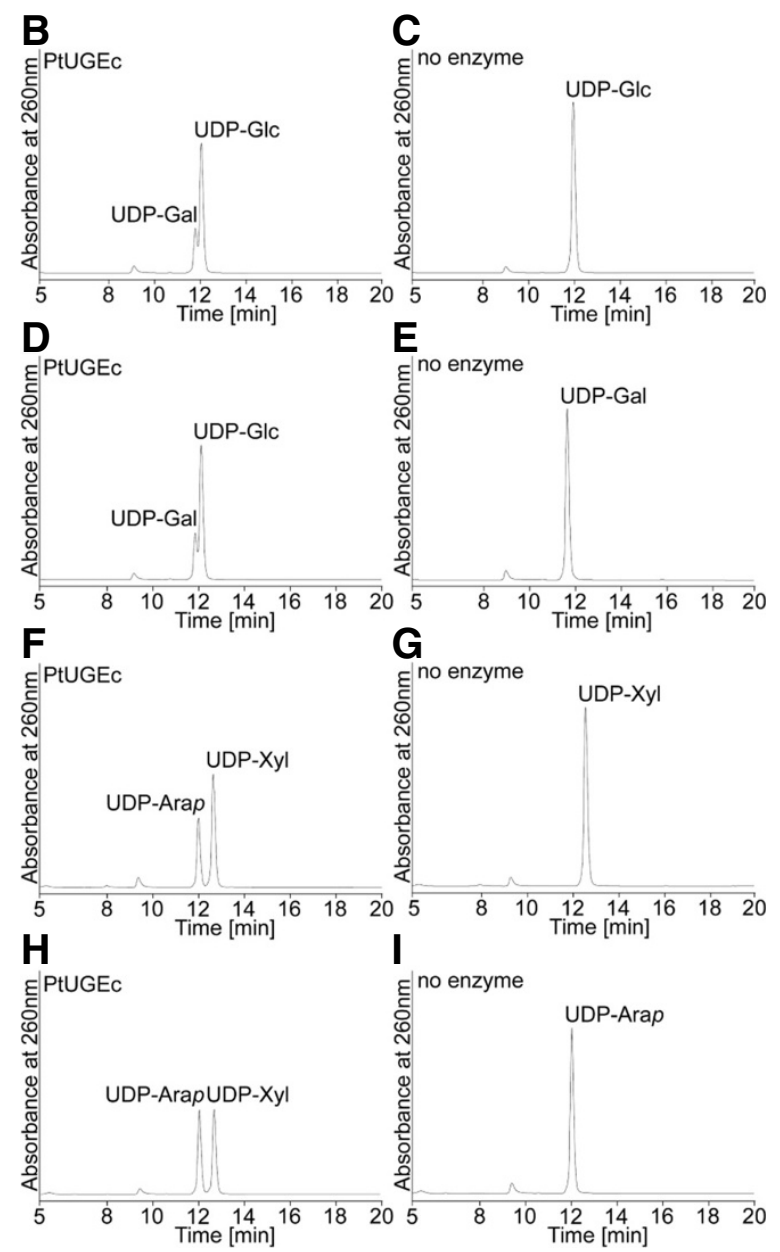

Figure 2 UDP-glucose 4-epimerase activity of recombinant PtUGEC protein. (A) SDS-PAGE analysis of different fractions from the purification of His-PtUGEC. (B-I) High-performance liquid chromatography (HPLC) chromatograms of nucleotide sugars incubated for 30 min with (left panels) or without (right panels) recombinant PtUGEc protein. and UDP-Gal, as well as UDP-Xyl and UDP-Ara (Figure 2B). The enzymatic reaction does not require the addition of $\mathrm{NAD}^{+}$, most likely because of noncovalent binding and co-purification of $\mathrm{NAD}^{+}$together with the enzyme, as it has been shown for the barley $H \nu$ UGE1 [17]. Final UDP-Gal and UDP-Glc concentrations were the same in reactions starting with UDP-Gal or with UDP-Glc, indicating that equilibrium levels were reached. The reactions starting with either UDP-Xyl or UDP-Ara did not completely reach the equilibrium level, suggesting that the reaction rates for UDP-Xyl/UDP-Ara interconversion are slower than for UDP-Gal/UDP-Glc. The bifunctional enzymatic characteristics of PtUGEc were somewhat unexpected for a UGE belonging to Clade II since other UGEs from this clade have been found to be specific for UDP-Glc/UDPGal interconversion. However, structural features that determine bifunctionality vs. mono-functionality are not known yet.

\section{All UGE constructs complement the UGE4 ${ }^{\text {rhd } 1}$ phenotype} In order to verify the functionality of UGE constructs, PtUGEc and the Arabidopsis AtUGE2, AtUGE4, and $A t$ UGE5 proteins were overexpressed under the control of the constitutive cauliflower mosaic virus $35 \mathrm{~S}$ promoter in the Arabidopsis uge4 mutant background. Loss of function of UGE4 results in a reduced root elongation rate and swelling of root epidermal cells probably as a result of defective cell wall matrix carbohydrate biosynthesis $[12,18]$. Thus, a simple visual screen can confirm complementation of the wild-type phenotype and thereby not only expression but also functionality of the UGE proteins. The four different UGE constructs all suppressed the root epidermal swelling and the reduced root length confirming previous UGE complementation results published by Barber et al. [9] and demonstrating that the poplar PtUGEc is functional in planta (Figure 3). The slight root length decrease in our UGE overexpressor plants as compared to wild type indicates however, that complementation is not complete. Expression of AtUGE2 and AtUGE5 resulted in almost complete complementation.

\section{Plants overexpressing UGE show no increase in cell wall bound galactose in leaf or stem cell walls}

All AtUGE2, AtUGE4, AtUGE5, or PtUGEc overexpressing Arabidopsis plants had a growth phenotype similar to wild-type Col- 0 and the empty vector control plants. The monosaccharide composition of non-cellulosic polysaccharides from leaves and stems was analyzed by highperformance anion exchange chromatography with pulsed amperometric detection (HPAEC-PAD). Two to four independent transgenic lines were analyzed for each construct in the T2 generation. Data for one representative line for each construct is shown in Figure 4. The 


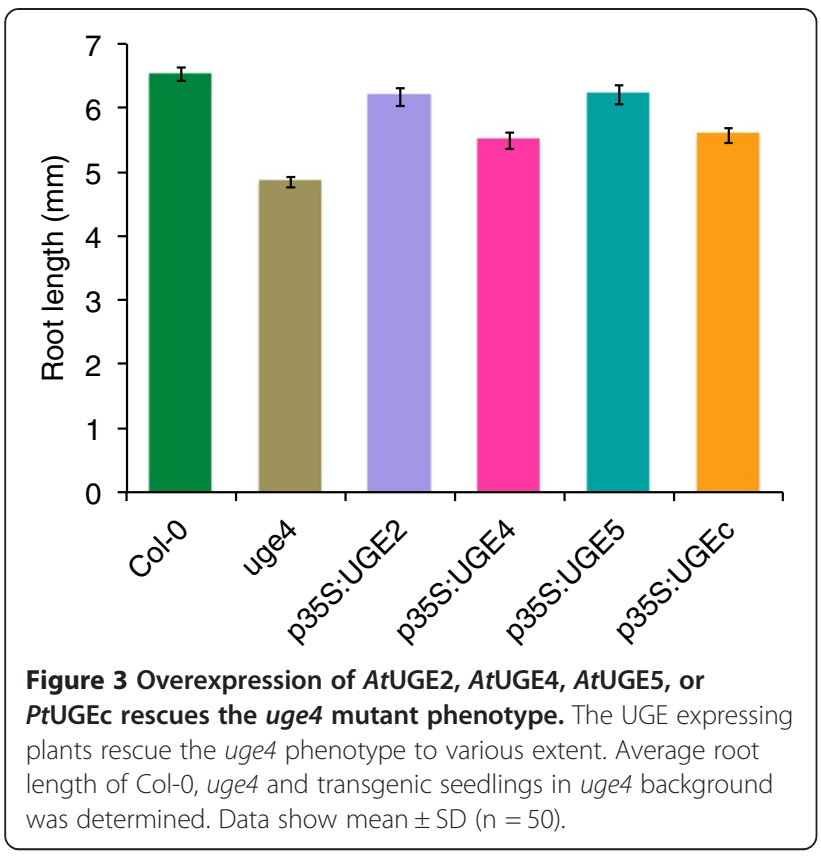

transgenic Arabidopsis UGE lines had no significant changes in sugar composition compared to the empty vector control plants in leaf cell walls (Figure 4A) or in stem cell walls (Figure 4B).

Recently, we have shown that overexpression of GalS1 in planta can lead to a significant $40 \%$ increase in total cell wall galactose in leaves [5]. Thus, the UDP-Gal substrate does not seem to be limiting for galactose incorporation into the cell wall in leaves and therefore an increase in interconverting UGE enzymes is not necessarily expected to lead to an increase in cell wall galactan.

\section{Cell wall bound galactose levels are increased in stem cell walls of co-overexpressers}

Since overexpression of AtUGE2, AtUGE4, AtUGE5, or PtUGEc alone did not result in a significant increase of total galactose in stems, we designed a gene stacking approach by co-expressing AtUGE2 together with the galactan synthase GalS1. AtUGE2 was chosen because it showed efficient complementation of the uge4 root phenotype (Figure 3). Although we had initially preferred to use a poplar UGE, PtUGEc was not a good choice because of its bispecificity and incomplete ability to complement uge4. We designed two different constructs for AtUGE2 expression. One under the control of the constitutive $35 \mathrm{~S}$ promoter ( $p 35 S: U G E 2)$ and a second fiber-specific construct (pIRX5:NST1-UGE2). The fiber-specific construct is under control of the IRX5 promoter and in addition expresses the transcription factor NST1 leading to a positive-artificial feedback loop and increased wall thickness in fiber cells, as previously reported [2]. In this construct UGE2 is expressed from the same PIRX5 promoter, separated from NST1 with the $2 \mathrm{~A}$ sequence from foot-and-mouth disease virus allowing coordinate expression of multiple proteins [19]. The stem cell wall composition was analyzed in the T2 generation for three independent lines for each construct. For each construct there was no difference between the independent lines and one line was selected for confirmation of the results in the T3 generation (Figure 5). Expression of p35S:UGE2 and pIRX5:NST1-UGE2, respectively, in the background of plants constitutively overexpressing GalS1 (p35S:GalS1) led to significantly increased galactose levels $(\mathrm{p} \leq 0.01)$ as shown by analysis of the monosaccharide composition of cell walls from stems (Figure 5). While $p 35 S$ : UGE2/p35S:GalS1 plants showed a galactose increase of more than 80\%, the pIRX5:NST1-UGE2/p35S:GalS1 galactose levels were only increased by $44 \%$ as compared to empty vector control (EVC) plants (Figure 5). Plants expressing p35S:GalS1 alone had only a slight increase in stem wall bound galactose, and no galactose increase was observed when the PIRX5:NST1-UGE2 construct was incorporated in the wild-type background. The apparent increase in xylose in plants containing the pIRX5:NST1-UGE2 construct for overexpression of the feedback-loop construct with NST1 could be expected in plants with increased fiber cell wall density and more xylan. However, the xylose content in these plants is not significantly different from the control ( $p>0.05$ even without Holm-Bonferroni correction). These results show that GalS1 is limiting for galactan synthesis in both leaves and stems while UGEs are not. However, when GalS1 is overexpressed, AtUGE2 or other UGEs seem to become limiting for how much galactan can be accumulated in cell walls of Arabidopsis plant stems. The $80 \%$ increase in cell wall galactose may not be the limit for what can be achieved. UDP-Gal formed by UGE2 must be transported into the Golgi lumen to be used by GalS1, and it is possible that the transport becomes limiting when both UGE2 and GalS1 are overexpressed. Recently, a UDP-Gal transporter URGT1 has been characterized, the overexpression of which results in increased cell wall galactan in leaves [20]. We are currently investigating the effect of overexpressing the transporter together with UGE2 and GalS1.

The increase in galactose of $p 35 S: U G E 2 / p 35 S: G a l S 1$ and pIRX5:NST1-UGE2/p35S:GalS1 co-overexpressor plants was further investigated by immunofluorescence microscopy of stem sections (Figure 6). Although immunofluorescence microscopy is not easily quantified, the detection of the LM5 galactan epitope was strongly increased specifically in the secondary cell walls of top and bottom stem sections of p35S:UGE2/p35S:GalS1 and pIRX5:NST1-UGE2/ p35S:GalS1 co-overexpressors, as compared to overexpressor lines of p35S:GalS1 or pIRX5:NST1-UGE2 alone, or the empty vector control. The fiber-specific pIRX5 constructs in addition resulted in highly thickened cell walls as an 

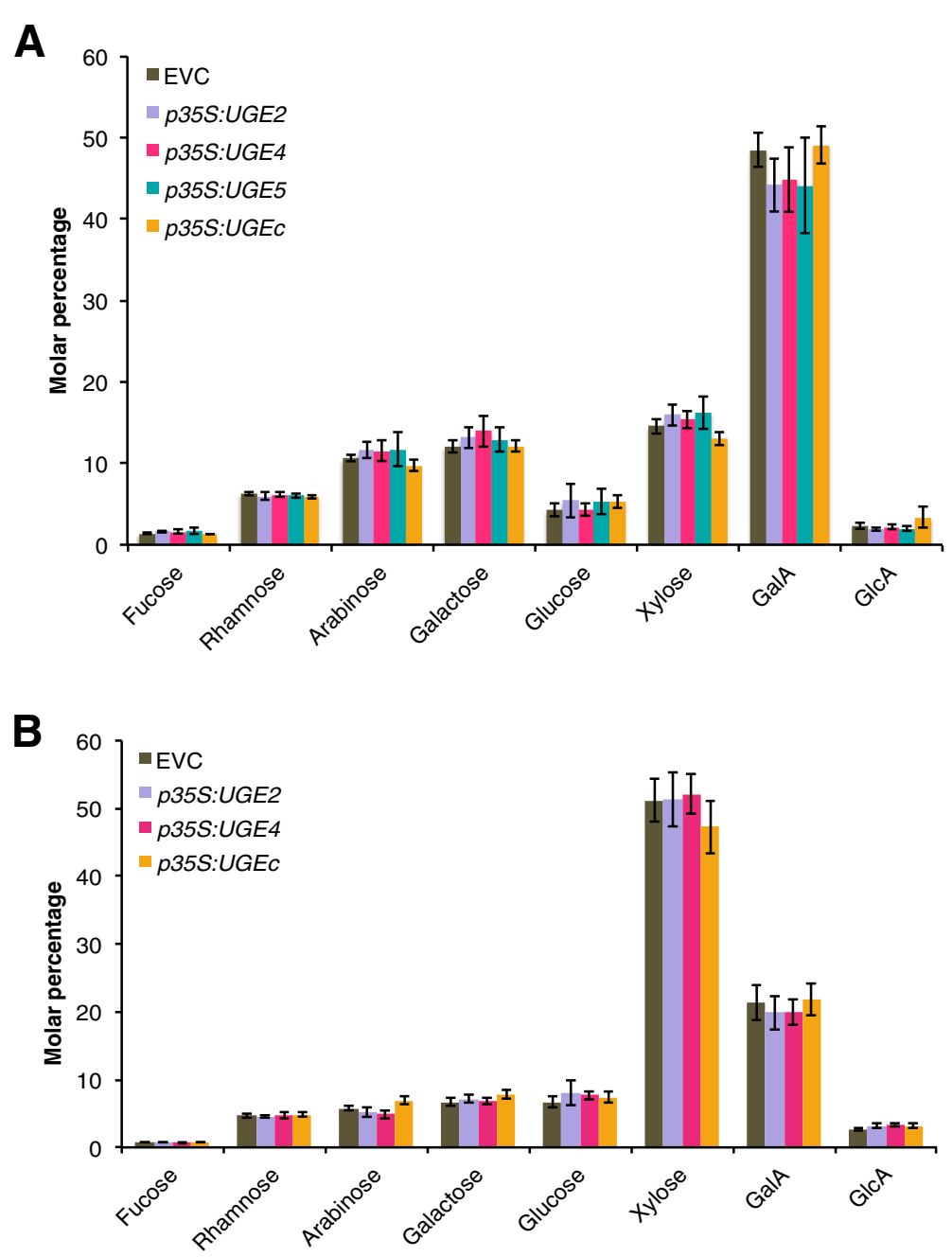

Figure 4 Cell wall monosaccharide composition analysis of leaf (A) and stem (B) material from UGE overexpression plants. Monosaccharide composition analysis of leaf (A) and stem (B) cell walls of plants constitutively overexpressing AtUGE2, AtUGE4, AtUGE5 did not show any significant differences compared to the empty vector control (EVC) ( $p>0.05$, pairwise t-test with Holm-Bonferroni correction for family-wise error rate). Monosaccharide levels are shown as molar percentage $\pm S D(n=6)$. GalA, a-D-galacturonic acid; GlcA, a-D-glucuronic acid.

effect of overexpressing NST1 under the IRX5 promoter and as visualized by lignin autofluorescence using a confocal microscope (Figure 6). On the one hand this increase in biomass density is highly desirable to improve the costeffectiveness of lignocellulosic bioenergy production. On the other hand however, the increase in xylan rather results in more recalcitrance and is counteracting the increase in the $\mathrm{C6} / \mathrm{C} 5$ sugar ratio, which is obtained due to increased galactan deposition. Therefore, future implementation of galactan overexpression could be improved by simultaneously downregulating xylan deposition in fiber cells. This could be achieved by using mutants deficient in xylan that have been complemented by reintroducing xylan biosynthesis specifically into the xylem vessels in order to restore wild type-like growth of the plants, as recently described
[3]. Conceivably, the lignin content in fiber cells also needs to be decreased.

\section{Conclusions}

Rescuing of the uge 4 root phenotype proved functionality of the constitutively expressed poplar $p 35 S: U G E c$ and the three Arabidopsis p35S:UGE2, p35S:UGE4 and p35S: $U G E 5$ constructs. Overexpression of any of the four UGE proteins alone did not increase the total cell wall galactose content in Arabidopsis leaves or stems. However, our gene stacking approach, combining overexpression of AtUGE2 and GalS1, clearly showed that it is possible to engineer plants with an even higher galactose content than GalS1 overexpressing plants by combining the overexpression of multiple genes into one plant. The 


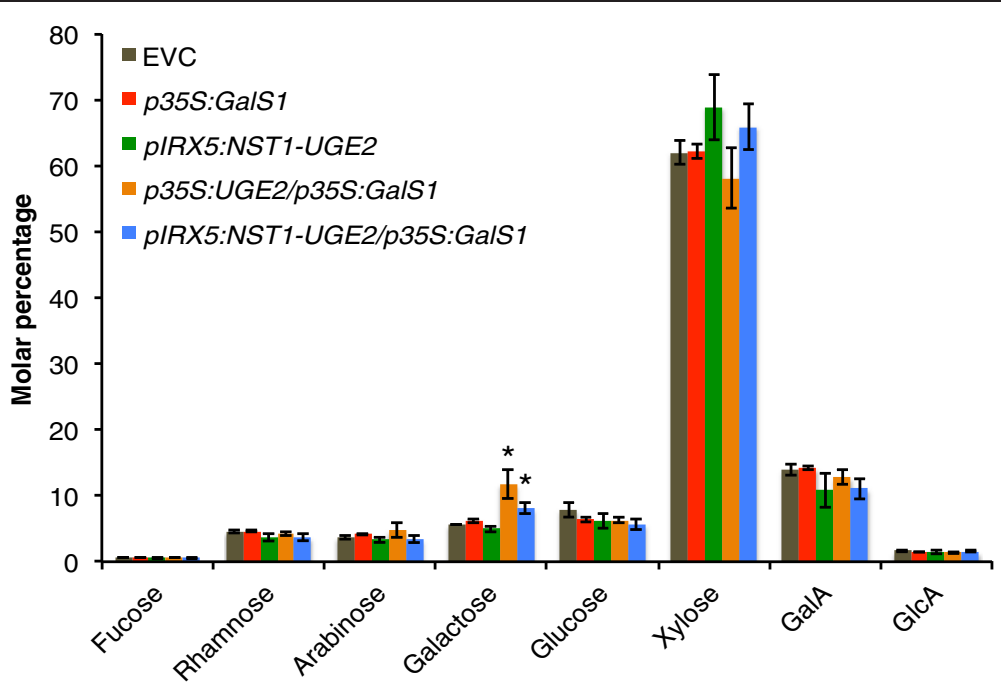

Figure 5 Cell wall monosaccharide composition analysis of stems from plants co-overexpressing AtUGE2 and GalS1. The monosaccharide composition of stems from plants in the T3 generation expressing either p35S:GalS1 or pIRX5:NST1-UGE2 or co-overexpressing either pIRX5:NST1-UGE2/p35S: GalS1 or p35S:UGE2/p35S:GalS1 was determined. Monosaccharide levels are shown as molar percentage \pm SD $(n=5)$. Significantly increased levels of galactose ( $p \leq 0.01$, indicated with asterisks) were found with the two co-expressing constructs pIRX5:NST1-UGE2/p35S:GalS1 and p35S:UGE2/p35S:GalS1, while no other sugars were different from empty vector control plants (EVC) in any of the transgenic plants $(p>0.05)$. Analysis in the T2 generation of three independent lines for each construct showed the same results. The data were analyzed by pairwise t-test with Holm-Bonferroni correction for family-wise error rate. GalA, a-D-galacturonic acid; GlcA, a-D-glucuronic acid.

Arabidopsis plants obtained have a more than $80 \%$ increase in stem galactose levels as compared to wildtype or empty vector control plants. Importantly, these transgenic plants exhibit no impairment of growth and development. Our study shows the promise of the gene stacking approach for engineering plants with improved properties for biofuel applications.

\section{Methods}

\section{Phylogenetic analysis}

Phylogenetic analyses were conducted in MEGA6 [21]. The bootstrap consensus tree inferred from 1000 replicates was taken to represent the evolutionary history of the taxa analyzed using the Neighbor-Joining method. The percentages of replicate trees in which the associated taxa clustered together in the bootstrap test are shown next to the branches retaining only groups with a frequency $\geq 60 \%$. Species and Genbank accession numbers are: An, Aspergillus niger [XP_001401007]; At, Arabidopsis thaliana [AEE28928, UGE1; AEE84827, UGE2; AEE 34065, UGE3; AEE34241, UGE4; AEE82951, UGE5]; Ao, Aspergillus oryzae [XP_001827449]; Bc, Bacillus cereus [ZP_01180393]; Bs, Bacillus subtilis [P55180]; Cl, Canis lupus [XP_544499]; Cr, Chlamydomonas reinhardtii [XP_001698706]; Ct, Cyamosis tetragonoloba [O65781]; Dd, Dictyostelium discoideum [XP_643834]; Dp, Drosohila pseudoobscura [XP_001352806]; Dr, Danio rerio [NP_00 1035389]; Gg, Gallus gallus [XP_417833]; Hs, Homo sapiens [Q14376]; Hv, Hordeum vulgare [AAX49504, UGE1; AAX49505, UGE2; AAX49503, UGE3]; Mt, Medicago truncatula [ACJ85116, UGE1; ACJ84690, UGE2]; Mxd, Malus $x$ domestica [BAF51705]; Ol, Ostreococcus lucimarinus [XP_001419325]; Os, Oryza sativa [BAF18426, UGE1; BAF23582, UGE2; BAF25641, UGE3; BAF24783, UGE4]; Ot, Ostreococcus tauri [CAL54894]; Pp, Physcomitrella patens [XP_001768301, UGE1; XP_001777464, UG E2; XP_001775163, UGE3; XP_001751529, UGE4; XP_00 1771084, UGE5]; Ps, Pisum sativum [AB381885]; Pt, Populus trichocarpa [XP_002304478, UGEa: XP_00230 3653, UGEb; XP_002299469, UGEc;] Pv, Paspalum vaginatum [BAE92559]; Sg, Streptococcus gordonii [AAN64 559]; St, Solanum tuberosum [AAP42567, UGE45; AAP9 7493, UGE51]; Sp, Streptococcus pneumonia [ZP_0182 5231]; Tc, Tribolium castaneum [XP_968616]; Xl, Xenopus laevis [NP_001080902]; Zm, Zea mays [AAP68981].

\section{Plant material and vectors}

All Arabidopsis thaliana (L.) Heynh. wild-type and mutant plant lines used were of ecotype Columbia-0 (Col-0). The AtUGE4 mutant uge4/rhd1-1 (At1g64440, CS2257) was obtained from the Arabidopsis Biological Resource Center (ABRC, http://www.arabidopsis.org). Plants overexpressing YFP-GalS1 (p35S:GalS1) have been previously described [5]. Populus trichocarpa Nisqually-1 leaf tissue was kindly donated by Dr. Lee Gunter (Oak Ridge National Laboratory). Entry vectors containing AtUGE2, AtUGE4 and AtUGE5 cDNA (At4g23920, At1g64440, At4g10960) and the Gateway-compatible plant transformation vectors pMDC32 and pMDC43 were obtained from ABRC. The destination vector pTKan-pIRX5-GWR3R2 


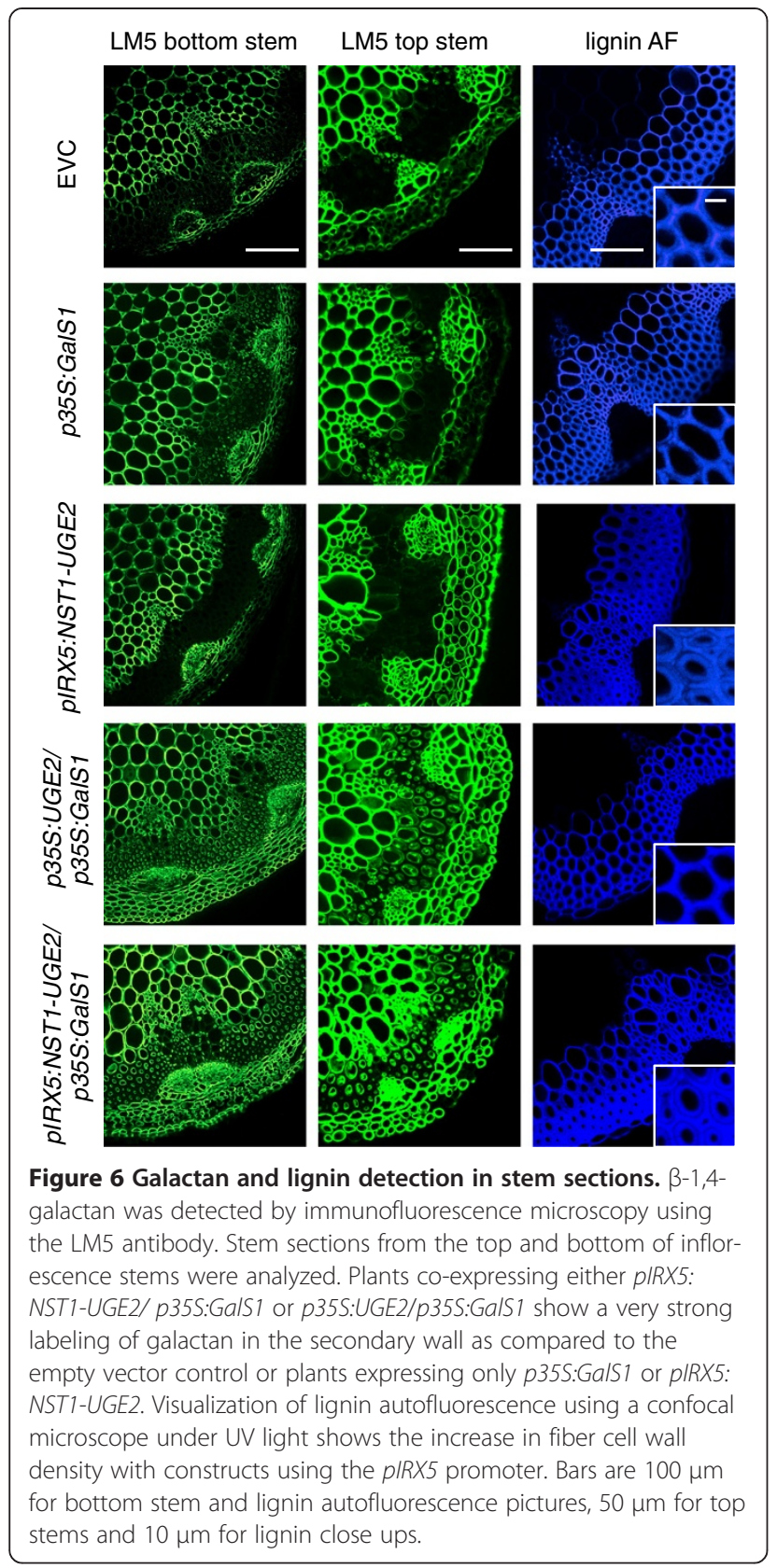

was made as described [1] except that it had attR2 and attR3 recombination sites. Gene constructs used to generate transgenic plants are shown in Figure 7.

\section{Generation of vectors and transformation of plants}

Flag-tagged UGE constructs were generated by PCR using Phusion Polymerase (Thermo Scientific) with primers fU2-F, fU2-R (AtUGE2); fU4-F, fU4-R (AtUGE4); fU5-F, fU5-R (AtUGE5); and fUc-F, fUc-R (PtUGEc) (Table 1), and cloned into vectors pMDC32 and pMDC43 using Gateway technology (Life Technologies). The fiber expressed AtUGE2 construct was generated in 2 steps. The sequence encoding for the NST1-2A-AtUGE2 fusion protein was first assembled in

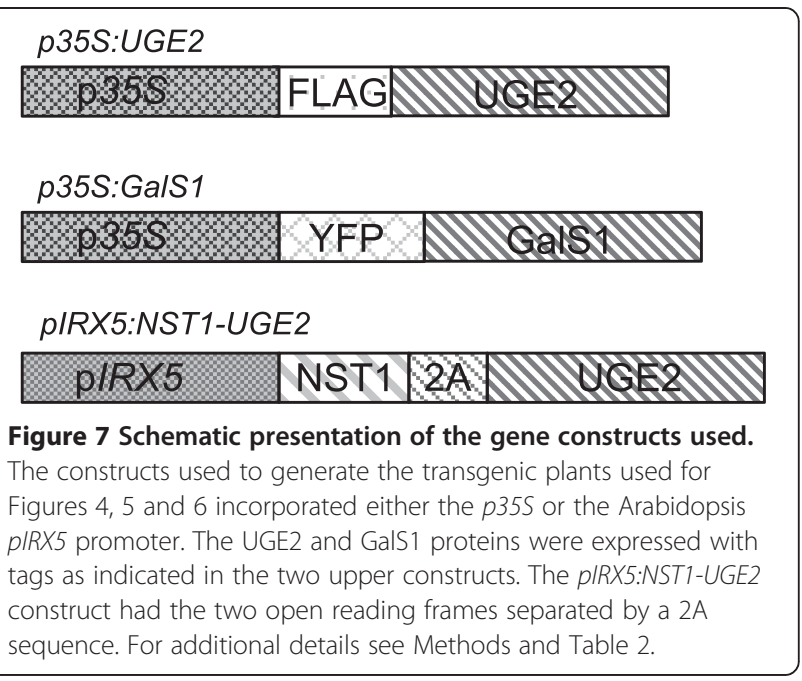

a gateway pDON-P3P2 vector (Life Sciences) to generate pDON-NST1-2A-AtUGE2-L3L2 plasmid. pDON-NST12A-AtUGE2-L3L2 vector was built using In-Fusion HD Cloning System (Clontech) to assemble PCR products of AtUGE2 and pDON-L3L2 vector containing the encoding sequence of NST1-2A (synthesized by GenScript, Piscatway, NJ) such that the encoding sequence of AtUGE2 was inserted in frame with the 3'end of NST1$2 \mathrm{~A}$ and at $5^{\prime}$ end of the attL2 sequences. AtUGE2 and pDON-NST1-2A-L3L2 PCR products were generated using Phusion Polymerase with ntU2-F/ntU2-R primer pair and F-pDON-attL2/R-pDON-NST1-2A primer pair respectively. The NST1-2A-AtUGE2 gene fusion was then transferred from into pTKan-pIRX5-GWR3R2 by LR recombination (Life technologies) to express NST1-2AAtUGE2 under the control of the fiber-specific pIRX5 promoter. PtUGEc cDNA was generated from RNA isolated from Poplar leaf tissue. RNA was extracted using the RNeasy Plant Minikit (Qiagen), treated with DNase I (Sigma) and cDNA was generated using iScript ${ }^{\mathrm{tm}}$ Reverse Transcription Supermix (Biorad). The cloned UGE open reading frames were confirmed by sequencing to be identical to the published sequences (see Figure 1 for accession numbers). For stable transformation of $A$. thaliana wild-type Columbia-0, uge4/rhd1-1 mutants or GalS1 overexpressing lines, constructs were transformed into $A$. tumefaciens strain C58-1 pGV3850 and plants were transformed using the floral dip method [22] (Table 2). Transformants were selected on MS medium containing hygromycin and transferred to soil. Plants confirmed to express the transgene were propagated and further characterized in the $\mathrm{T} 2$ and $\mathrm{T} 3$ generations.

\section{Plant growth conditions and measurements}

All plants were grown in a $14-16 \mathrm{~h}$ photoperiod at $120 \mu \mathrm{mol} \mathrm{m} \mathrm{m}^{-2} \mathrm{~s}^{-1}$ photon flux density. Plants for root 
Table 1 Primer sequences used in this work

\begin{tabular}{|c|c|}
\hline Primer name & Primer sequence \\
\hline fU2-F & 5'-CACCATGGATTACAAGGATGACGATGACAAGGCGAAGAGTG-3' \\
\hline fU2-R & 5'-TTATGAAGAGGAGCCATTGGAGGAGGA-3' \\
\hline fU4-F & 5'-CACCATGGATTACAAGGATGACGATGACAAGGTTGGGAATATT-3' \\
\hline$f \cup 4-R$ & 5'-TTATGTTGAGTTTGGTGAAGAACCGTAACC-3' \\
\hline$f \cup 5-F$ & 5'-CACCATGGATTACAAGGATGACGATGACAAGATGGCTAGAAACGT-3' \\
\hline fU5-R & 5'-TTAATGAGAGTTGTCTTCAGAAGAGG-3' \\
\hline fUc-F & 5'-CACCATGGATTACAAGGATGACGATGACAAGATGGCCTATAATATTC TGGTTACCG-3' \\
\hline fUc-R & 5'-TCAGTTTGTGCCGTCAGGAGATC-3' \\
\hline$n+U 2-F$ & 5'-TCTAATCCAGGACCTATGGCGAAGAGTGTITTGGTTAC-3' \\
\hline$n+U 2-R$ & 5'-CAAGAAAGCTGGGTCTGAAGAGGAGCCATTGGAGGAG-3' \\
\hline F-pDON-attL2 & 5'-GACCCAGCTTTCTTGTACAAAGT-3' \\
\hline R-pDON-NST1-2A & 5'-AGGTCCTGGATTAGACTCAACG-3' \\
\hline
\end{tabular}

length measurements were grown vertically on MS plates and scanned at 6 days after germination. The root length of about 50 individual seedlings was measured using ImageJ [23].

\section{Monosaccharide composition analysis of cell walls}

Bottom sections (5 cm length of stems) or whole leaves from 5-week-old plants were ground in liquid nitrogen and alcohol-insoluble residue (AIR) was prepared and enzymatically destarched as described [24]. Destarched AIR samples (1 mg) were hydrolyzed with $2 \mathrm{M}$ triflouroacetic acid (TFA) and monosaccharide composition was determined by HPAEC-PAD as described $[25,26]$.

\section{Expression and purification of His-PtUGEc}

PtUGEc was introduced into pDEST17 expression vector (Invitrogen) containing an N-terminal 6xHis-tag and an IPTG inducible promoter. Gene expression in BL21 Star cells (Invitrogen) was induced by adding IPTG to a final concentration of $1 \mathrm{mM}$, and cultures were grown at $18^{\circ} \mathrm{C}$ overnight. PtUGEc was purified from the supernatant of lysed cell pellets using HIS-Select Nickel Affinity Gel purification (Sigma-Aldrich). Lysis of bacterial cells took place using CelLytic B 2X containing $0.2 \mathrm{mg} / \mathrm{ml}$ lysozyme, $50 \mathrm{U} / \mathrm{ml}$ benzonase (all Sigma-Aldrich) and proteinase inhibitor cocktail (Roche). HIS-tagged PtUGEc was desalted with PD-10 desalting columns (Amersham Biosciences). Samples of $30 \mu \mathrm{g}$ His-PtUGEc protein were separated on a Novex 8-16\% Tris Glycine Gradient gel (Invitrogen) and stained with Coomassie Brilliant Blue.

\section{His-PtUGEc activity assay}

$5 \mu \mathrm{g}$ of purified His-PtUGEc was mixed with $1 \mathrm{mM}$ UDPglucose in $50 \mathrm{mM}$ Tris- $\mathrm{HCl}(\mathrm{pH}$ 8.0), $1 \mathrm{mM}$ DTT for $30 \mathrm{~min}$ at $30^{\circ} \mathrm{C}$. Reactions were terminated by incubating for $10 \mathrm{~min}$ at $90^{\circ} \mathrm{C}$ and filtration through $0.45 \mu \mathrm{m}$ filters (Millipore) prior to quantification. To test more nucleotide sugar substrates, the same reaction mixture as described above was used, substituting UDP-Glc with UDP-Gal, UDP-Xyl or UDP-Ara. Negative controls of purified protein boiled for $10 \mathrm{~min}$ at $90^{\circ} \mathrm{C}$ were used for all reactions. Separation and quantification of UDP-sugars in terminated reactions was performed by HPAEC analysis using a Dionex Ultimate 3000 system (Thermo Fisher) with detection at $262 \mathrm{~nm}$. Samples were separated on a CarboPac PA20 column (Thermo Fisher) and eluted with an ammonium formate gradient according to Rautengarten et al. [27]. Standard solutions containing UDP-Glc, UDPGal (both Sigma Aldrich), UDP-Ara and UDP-Xyl (both Carbosource Service) were run as references.

Table 2 Constructs used for plant expression

\begin{tabular}{lll}
\hline Name & Construct & Expression vector \\
\hline p35S:UGE2 & p35S:FLAG-AtUGE2 & pMDC32 \\
p35S:UGE4 & p35S:FLAG-AtUGE4 & pMDC32 \\
p35S:UGE5 & p35S:FLAG-AtUGE5 & pMDC32 \\
p35S:UGEC & p35S:FLAG-PtUGEC & pMDC32 \\
p35S:GalS1 & p35S:YFP-GalS1 & pEarleyGate104 \\
pIRX5:NST1-UGE2 & pIRX5:NST1-2A-AtUGE2 & pTKan-plRX5-GWR3R2 \\
EVC & 35S:pvu2 (non coding) & pMDC32 \\
\hline
\end{tabular}




\section{Immunofluorence microscopy}

The top and base $3 \mathrm{~cm}$ of main stems from 6-week-old plants (3 stems/line) were harvested and fixed overnight at $4^{\circ} \mathrm{C}$ in fixative solution (4\% paraformaldehyde in $50 \mathrm{mM}$ piperazine-N-N'-bis(2-ethanesulphonic acid), 5 mM EGTA, $\mathrm{pH}$ 6.9). Fixed stem sections were embedded in $7 \%$ agarose and sectioned using a Leica VT1000S vibratome. Stem sections were labeled with monoclonal LM5 rabbit antibody (PlantProbes, Leeds, UK), which recognizes 1,4linked $\beta$-galactan [28]. The labeling was performed according to Verhertbruggen et al. [29]. Sections were mounted on slides and pictures were taken using a LSM 710 confocal Microscope (Carl Zeiss). Lignin autofluorescence was monitored using a $405 \mathrm{~nm}$ Diode laser. Images were acquired with the Zen software package (Carl Zeiss) and analyzed with ImageJ [23].

\section{Abbreviations \\ GalA: a-D-galacturonic acid; GlcA: a-D-glucuronic acid; GalS1: GALACTAN SYNTHASE1; GT: Glycosyltransferase; NST: NAC secondary wall thickening promoting factor; NAC: NAM ATAF1/2 and CUC2; UGE: UDP-glucose 4-epimerase; UDP: Uridine diphosphate.}

\section{Competing interests}

Parts of the strategy described in this paper have been included in a patent application with HVS as inventor. The remaining authors declare that they have no competing interests.

\section{Authors' contributions}

HVS designed and coordinated the study; VMG, RS, AJML, BE, DL and CR conducted the experiments; VMG, RS, BE, CR and HVS conducted data analysis; VMG, RS and HVS wrote the manuscript, and all authors read and approved the final manuscript.

\section{Acknowledgements \\ This work conducted by the Joint BioEnergy Institute was supported by the Office of Science, Office of Biological and Environmental Research, through contract DE-AC02-05CH11231 between Lawrence Berkeley National Laboratory and the U. S. Department of Energy. RS was supported by a research fellowship of the German Research Foundation (Deutsche Forschungsgemeinschaft). Funding for BE was provided by the Danish Council for Strategic Research. We thank Ms. Sherry Chan for assistance with plant growth and Dr. Lee Gunter for providing poplar material for CDNA purification.}

\section{Author details}

${ }^{1}$ Feedstocks Division, Joint BioEnergy Institute, Emeryville, California 94608, USA. ${ }^{2}$ Physical Biosciences Division, Lawrence Berkeley National Laboratory, Berkeley, California 94720, USA. ${ }^{3}$ Department of Plant and Environmental Sciences, University of Copenhagen, DK-1871 Frederiksberg C, Denmark. ${ }^{4}$ Department of Plant and Microbial Biology, University of California, Berkeley, California 94720, USA.

Received: 10 September 2014 Accepted: 20 November 2014 Published online: 10 December 2014

\section{References}

1. Eudes A, George A, Mukerjee P, Kim JS, Pollet B, Benke PI, Yang F, Mitra P, Sun L, Cetinkol OP, Chabout S, Mouille G, Soubigou-Taconnat L, Balzergue S, Singh S, Holmes BM, Mukhopadhyay A, Keasling JD, Simmons BA, Lapierre C, Ralph J, Loque D: Biosynthesis and incorporation of side-chaintruncated lignin monomers to reduce lignin polymerization and enhance saccharification. Plant Biotechnol J 2012, 10:609-620.

2. Yang F, Mitra P, Zhang L, Prak L, Verhertbruggen Y, Kim JS, Sun L, Zheng K, Tang K, Auer M, Scheller HV, Loque D: Engineering secondary cell wall deposition in plants. Plant Biotechnol J 2013, 11:325-335.
3. Petersen PD, Lau J, Ebert B, Yang F, Verhertbruggen Y, Kim JS, Varanasi $P$, Suttangkakul A, Auer M, Loque D, Scheller HV: Engineering of plants with improved properties as biofuels feedstocks by vessel-specific complementation of xylan biosynthesis mutants. Biotechnol Biofuels 2012, $5: 84$.

4. Lairson LL, Henrissat B, Davies GJ, Withers SG: Glycosyltransferases: structures, functions, and mechanisms. Annu Rev Biochem 2008, 77:521-555.

5. Liwanag AJ, Ebert B, Verhertbruggen Y, Rennie EA, Rautengarten C, Oikawa A, Andersen MC, Clausen MH, Scheller HV: Pectin biosynthesis: GALS1 in Arabidopsis thaliana is a beta-1,4-galactan beta-1,4-galactosyltransferase. Plant Cell 2012, 24:5024-5036.

6. Bar-Peled M, O'Neill MA: Plant nucleotide sugar formation, interconversion, and salvage by sugar recycling. Annu Rev Plant Biol 2011, 62:127-155.

7. Reboul R, Geserick C, Pabst M, Frey B, Wittmann D, Lutz-Meindl U, Leonard $\mathrm{R}$, Tenhaken R: Down-regulation of UDP-glucuronic acid biosynthesis leads to swollen plant cell walls and severe developmental defects associated with changes in pectic polysaccharides. J Biol Chem 2011 286:39982-39992.

8. Burget EG, Verma R, Molhoj M, Reiter WD: The biosynthesis of L-arabinose in plants: molecular cloning and characterization of a Golgi-localized UDP-D-xylose 4-epimerase encoded by the MUR4 gene of Arabidopsis. Plant Cell 2003, 15:523-531.

9. Barber C, Rosti J, Rawat A, Findlay K, Roberts K, Seifert GJ: Distinct properties of the five UDP-D-glucose/UDP-D-galactose 4-epimerase isoforms of Arabidopsis thaliana. J Biol Chem 2006, 281:17276-17285.

10. Rösti J, Barton CJ, Albrecht S, Dupree P, Pauly M, Findlay K, Roberts K, Seifert GJ: UDP-glucose 4-epimerase isoforms UGE2 and UGE4 cooperate in providing UDP-galactose for cell wall biosynthesis and growth of Arabidopsis thaliana. Plant Cell 2007, 19:1565-1579.

11. Kotake T, Takata R, Verma R, Takaba M, Yamaguchi D, Orita T, Kaneko S, Matsuoka K, Koyama T, Reiter WD, Tsumuraya Y: Bifunctional cytosolic UDP-glucose 4-epimerases catalyse the interconversion between UDP-Dxylose and UDP-L-arabinose in plants. Biochem J 2009, 424:169-177.

12. Seifert GJ, Barber C, Wells B, Dolan L, Roberts K: Galactose biosynthesis in Arabidopsis: genetic evidence for substrate channeling from UDP-Dgalactose into cell wall polymers. Curr Biol 2002, 12:1840-1845.

13. Nguema-Ona E, Andeme-Onzighi C, Aboughe-Angone S, Bardor M, Ishii T, Lerouge $P$, Driouich A: The reb1-1 mutation of Arabidopsis. Effect on the structure and localization of galactose-containing cell wall polysaccharides. Plant Physiol 2006, 140:1406-1417.

14. Oomen RJFJ, Dao-Thi B, Tzitzikas EN, Bakx EJ, Schols HA, Visser RGF, Vincken JP: Overexpression of two different potato UDP-Glc 4-epimerases can increase the galactose content of potato tuber cell walls. Plant Science 2004, 166:1097-1104.

15. Taylor NG: Cellulose biosynthesis and deposition in higher plants. New Phytol 2008, 178:239-252.

16. Mitsuda N, Iwase A, Yamamoto H, Yoshida M, Seki M, Shinozaki K, Ohme-Takagi M: NAC transcription factors, NST1 and NST3, are key regulators of the formation of secondary walls in woody tissues of Arabidopsis. Plant Cell 2007, 19:270-280.

17. Zhang Q, Hrmova M, Shirley NJ, Lahnstein J, Fincher GB: Gene expression patterns and catalytic properties of UDP-D-glucose 4-epimerases from barley (Hordeum vulgare L.). Biochem J 2006, 394:115-124.

18. Andeme-Onzighi C, Sivaguru M, Judy-March J, Baskin TI, Driouich A: The reb1-1 mutation of Arabidopsis alters the morphology of trichoblasts, the expression of arabinogalactan-proteins and the organization of cortical microtubules. Planta 2002, 215:949-958.

19. Halpin C, Cooke SE, Barakate A, Amrani A, Ryan MD: Self-processing 2Apolyproteins - a system for co-ordinate expression of multiple proteins in transgenic plants. Plant J 1999, 17:453-459.

20. Rautengarten C, Ebert B, Moreno I, Temple H, Herter T, Link B, Doñas D, Moreno A, Saéz-Aguayo S, Blanco MF, Mortimer J, Schultink A, Reiter WD, Dupree P, Pauly M, Heazlewood JL, Scheller HV, Orellana A: The Golgi UDPRhamnose / UDP-Galactose transporter family in Arabidopsis. Proc Natl Acad Sci USA 2014, 111:11563-11568.

21. Tamura K, Stecher G, Peterson D, Filipski A, Kumar S: MEGA6: Molecular Evolutionary Genetics Analysis version 6.0. Mol Biol Evol 2013, 30:2725-2729.

22. Clough SJ, Bent AF: Floral dip: a simplified method for Agrobacteriummediated transformation of Arabidopsis thaliana. Plant J 1998, 16:735-743 
23. Abramoff MD, Magalhaes PJ, Ram SJ: Image processing with ImageJ. Biophotonics Int 2004, 11:36-42.

24. Harholt J, Jensen JK, Sorensen SO, Orfila C, Pauly M, Scheller HV: Arabinan deficient 1 is a putative arabinosyltransferase involved in biosynthesis of pectic arabinan in Arabidopsis. Plant Physiol 2006, 140:49-58.

25. Øbro J, Harholt J, Scheller HV, Orfila C: Rhamnogalacturonan I in Solanum tuberosum tubers contains complex arabinogalactan structures. Phytochemistry 2004, 65:1429-1438

26. Yin L, Verhertbruggen Y, Oikawa A, Manisseri C, Knierim B, Prak L, Jensen JK, Knox JP, Auer M, Willats WG, Scheller HV: The cooperative activities of CSLD2, CSLD3, and CSLD5 are required for normal Arabidopsis development. Mol Plant 2011, 4:1024-1037.

27. Rautengarten C, Ebert B, Herter T, Petzold CJ, Ishii T, Mukhopadhyay A, Usadel B, Scheller HV: The interconversion of UDP-arabinopyranose and UDP-arabinofuranose is indispensable for plant development in Arabidopsis. Plant Cell 2011, 4:1373-90.

28. Jones $L$, Seymour GB, Knox JP: Localization of pectic galactan in tomato cell walls using a monoclonal antibody specific to (1->4)-beta-Dgalactan. Plant Physiol 1997, 113:1405-1412.

29. Verhertbruggen Y, Marcus SE, Haeger A, Verhoef R, Schols HA, McCleary BV, McKee L, Gilbert HJ, Knox JP: Developmental complexity of arabinan polysaccharides and their processing in plant cell walls. Plant I 2009, 59:413-425

doi:10.1186/s12870-014-0344-x

Cite this article as: Gondolf et al:: A gene stacking approach leads to engineered plants with highly increased galactan levels in Arabidopsis. BMC Plant Biology 2014 14:344.

\section{Submit your next manuscript to BioMed Central} and take full advantage of:

- Convenient online submission

- Thorough peer review

- No space constraints or color figure charges

- Immediate publication on acceptance

- Inclusion in PubMed, CAS, Scopus and Google Scholar

- Research which is freely available for redistribution 